\title{
Hybrid method for determining the parameters of condenser microphones from measured membrane velocities and numerical calculations
}

\author{
Barrera Figueroa, Salvador; Rasmussen, Knud; Jacobsen, Finn
}

Published in:

Acoustical Society of America. Journal

Link to article, DOI:

$10.1121 / 1.3203939$

Publication date:

2009

Document Version

Publisher's PDF, also known as Version of record

Link back to DTU Orbit

Citation (APA):

Barrera Figueroa, S., Rasmussen, K., \& Jacobsen, F. (2009). Hybrid method for determining the parameters of condenser microphones from measured membrane velocities and numerical calculations. Acoustical Society of America. Journal, 126(4), 1788-1795. https://doi.org/10.1121/1.3203939

\section{General rights}

Copyright and moral rights for the publications made accessible in the public portal are retained by the authors and/or other copyright owners and it is a condition of accessing publications that users recognise and abide by the legal requirements associated with these rights.

- Users may download and print one copy of any publication from the public portal for the purpose of private study or research.

- You may not further distribute the material or use it for any profit-making activity or commercial gain

- You may freely distribute the URL identifying the publication in the public portal 


\title{
Hybrid method for determining the parameters of condenser microphones from measured membrane velocities and numerical calculations ${ }^{\text {a) }}$
}

\author{
Salvador Barrera-Figueroa ${ }^{\text {b) }}$ and Knud Rasmussen \\ Danish Primary Laboratory of Acoustics, Danish Fundamental Metrology Ltd., Matematiktorvet 307, 2800 \\ Kongens Lyngby, Denmark \\ Finn Jacobsen \\ Acoustic Technology, Department of Electrical Engineering, Technical University of Denmark, Ørsteds \\ Plads 352, 2800 Kgs. Lyngby, Denmark
}

(Received 27 February 2009; revised 15 June 2009; accepted 20 July 2009)

\begin{abstract}
Typically, numerical calculations of the pressure, free-field, and random-incidence response of a condenser microphone are carried out on the basis of an assumed displacement distribution of the diaphragm of the microphone; the conventional assumption is that the displacement follows a Bessel function. This assumption is probably valid at frequencies below the resonance frequency. However, at higher frequencies the movement of the membrane is heavily coupled with the damping of the air film between membrane and backplate and with resonances in the back chamber of the microphone. A solution to this problem is to measure the velocity distribution of the membrane by means of a non-contact method, such as laser vibrometry. The measured velocity distribution can be used together with a numerical formulation such as the boundary element method for estimating the microphone response and other parameters, e.g., the acoustic center. In this work, such a hybrid method is presented and examined. The velocity distributions of a number of condenser microphones have been determined using a laser vibrometer, and these measured velocity distributions have been used for estimating microphone responses and other parameters. The agreement with experimental data is generally good. The method can be used as an alternative for validating the parameters of the microphones determined by classical calibration techniques.
\end{abstract}

(C) 2009 Acoustical Society of America. [DOI: 10.1121/1.3203939]

PACS number(s): 43.38.Kb, 43.38.Bs [AJZ]

Pages: $1788-1795$

\section{INTRODUCTION}

The numerical calculation of pressure, free-field, and random-incidence responses of microphones has become a popular method for validating results obtained experimentally. Furthermore, numerical calculations are sometimes used to complement experimental results at frequencies where the experimental methods might yield unreliable results. ${ }^{1-5}$ However, the numerical calculations are usually carried out under a number of assumptions that are not necessarily completely realistic. Several attempts to develop a complete coupled model of a condenser microphone numerically are described in the literature. ${ }^{6-8}$ Unfortunately, determining the velocity of the membrane numerically has proven to be an elusive task. Whereas complex geometries and configurations can easily be simulated, other parameters such as the velocity distribution of the membrane of a microphone are usually assumed to have a well defined analytical form. However, experimental results indicate that the velocity of the membrane may have a quite different shape.

\footnotetext{
Portions of this work were presented in "On determination of microphone response and other parameters by a hybrid experimental and numerical method," Proceedings of Acoustics'08, Paris, France, June 2008.

b) Author to whom correspondence should be addressed. Electronic mail: sbf@dfm.dtu.dk
}

A few years ago, Behler and Vorländer proposed an alternative solution that consists of measuring the velocity of the membrane of the microphone using a non-contact method, laser vibrometry. The measured membrane velocity was used in determining the "monopole sensitivity" of the microphone (i.e., the pressure sensitivity) and also combined with a numerical model of the effect of the body of the microphone and used for determining the free-field sensitivity. ${ }^{9}$ However, in the authors' words, "The scope [of their investigation was] not to achieve maximum accuracy (...) but to illustrate whether the method is worth to be checked for qualification as precision method or not," and thus the results were not compared with precision data.

This paper pursues the same idea and examines the possibility of using the measured velocity of the membrane of a microphone for determining a number of microphone parameters. The velocity of the membrane of laboratory standard (LS) microphones has been measured using a laser vibrometer, and this velocity has been used in a boundary element method (BEM) model of a microphone as the boundary condition at the membrane of the microphone. The acoustic center, the free-field correction, the pressure sensitivities, and directivity index of several different types of microphones have been determined from the calculated pressure on the membrane and the sound field surrounding the microphone. 
a)

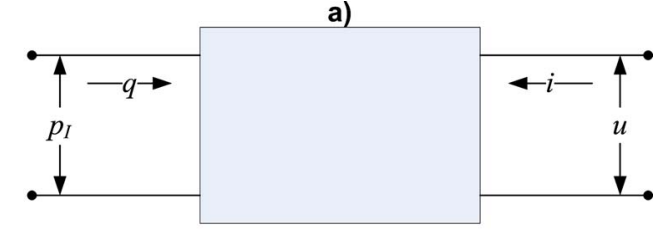

b)

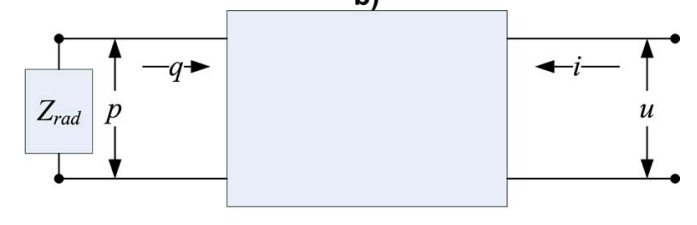

c)

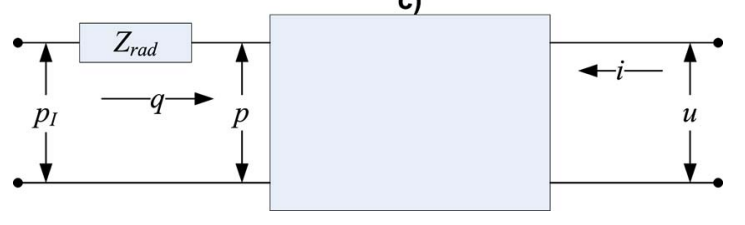

FIG. 1. (Color online) Network representation of a microphone. (a) Unloaded microphone, (b) microphone used as a sound source, and (c) microphone used as a receiver.

\section{THEORETICAL BACKGROUND}

A condenser microphone is a reciprocal transducer the behavior of which can be defined in terms of the equations of a four port electro-acoustic network. A graphic representation of the network of the microphone is shown in Fig. 1.

Because a condenser microphone is a reciprocal transducer, the open-circuit pressure sensitivity of the microphone, $M_{p}$, can be determined from the canonical equations of the network, ${ }^{10-12}$

$$
M_{p}=\frac{u_{i=0}}{p}=-\frac{q_{p=0}}{i},
$$

where $u_{i=0}$ is the open-circuit voltage (no electric load), $q_{p=0}$ is the volume velocity under conditions of no acoustic load, $p$ is the pressure on the acoustic terminal, and $i$ is the current. If the microphone acts as a sound source, the ratio of the volume velocity to the current will be affected by the load of the radiation impedance,

$$
-\frac{q}{i}=M_{p} \frac{Z_{a}}{Z_{a}+Z_{\mathrm{rad}}},
$$

where $Z_{a}$ is the acoustic impedance of the microphone and $Z_{\text {rad }}$ is the radiation impedance.

\section{A. Acoustic center}

The concept of acoustic center has been widely used in the development and practical realization of free-field reciprocity calibration of microphones. ${ }^{12-14}$ In this context, the microphone is substituted by a point source or a point receiver located at the position of the acoustic center. Thus, the accuracy of the estimated free-field sensitivity of a measurement microphone depends on the accuracy of the position of the acoustic center. In an international standard, the acoustic center of a microphone is defined as follows: "For a sound emitting transducer, for a sinusoidal signal of given fre- quency and for a specified direction and distance, the point from which the approximately spherical wavefronts, as observed in a small region around the observation point, appear to diverge." 12

The acoustic center of LS microphones has been determined using different methodologies: (a) from the decay of the sound pressure with the distance when a condenser microphone is used as a transmitter and the sound pressure was measured using a probe microphone, ${ }^{15}$ (b) from measurements of the transfer impedance between two microphones at different distances, ${ }^{3}$ and (c) from measuring the decay of the sound pressure with the distance when the sound field is generated by a source of known acoustic center and the decay of the sound pressure is measured with the microphone under test. $^{16}$

Method (a) provided values of the acoustic center that were in good agreement with the expected theoretical results, but there were significant variations due to reflections in the anechoic chamber. The acoustic centers determined using method (b) combined with a time-selective technique that removed the influence of reflections provided values of the acoustic center that were in good agreement with numerical BEM estimates. However, method (b) may not give reliable values at low frequencies because of the application of the time-selective procedure introduces some unwanted ripple at the extremes of the frequency range. Method (c) is designed to provide the acoustic center at low frequencies because the development is based on assumptions about the membrane of the source having a simple theoretical form.

The experimental values presented in Ref. 3 showed discrepancies at high frequencies with an estimate of the acoustic center determined numerically using BEM. It was concluded that such a discrepancy might be due to a possible difference between the simple analytical velocity distribution of the membrane of the microphone used in the BEM calculations and the actual velocity distribution of the membrane of the microphone. However, using the measured velocity distribution together with BEM implies that no theoretical guess of how the membrane moves is needed.

It can be assumed that the microphones can be regarded as axi-symmetric sources. Under this assumption, the acoustic center must be somewhere on the axis. If the amplitude of the sound pressure is plotted as a function of the distance, a straight line can be fitted over the region of concern. Thus, the position of the acoustic center, $x(k, r)$, can be determined using the expression

$$
x(k, r)=r+|p(r)| /(\partial|p(r)| / \partial r),
$$

where $k$ is the wave number, $r$ is the axial distance from the diaphragm of the microphone, $p(r)$ is the sound pressure as a function of distance, and the rate of change, $\partial|p(r)| / \partial r$, must be estimated by any available means, for example, by using least-squares fitting. ${ }^{3}$

\section{B. Free-field correction}

The free-field sensitivity of microphones can be determined directly for each microphone using either primary (reciprocity) or secondary (comparison) techniques. The ap- 
plication of these techniques requires either expensive experimental facilities such as an anechoic room or analysis techniques that simulate free-field conditions. This makes it difficult to determine the free-field sensitivity. A common alternative is to determine the free-field sensitivity as the sum of the pressure sensitivity (in decibel) and a free-field correction. ${ }^{17,18}$ The free-field correction, $C_{\mathrm{ff}}$, is defined as the logarithmic ratio of the free-field sensitivity to the pressure sensitivity,

$$
C_{\mathrm{ff}}=10 \log \left\{\left|M_{\mathrm{ff}}\right|^{2} /\left|M_{p}\right|^{2}\right\}
$$

where $M_{\mathrm{ff}}$ is the free-field sensitivity and $M_{p}$ is the pressure sensitivity of the microphone. The free-field correction can also be defined as the product of the free-field sensitivity (in linear units), the diffraction factor, and the load of the radiation impedance on the radiation impedance of the microphone. ${ }^{12}$ Therefore, the free-field correction will mainly depend on the geometry and acoustic impedance of the microphone. Thus, it remains unchanged if the microphone does not suffer from any change in its geometry or impedance. Additionally, for a particular type of microphone, the geometry and impedance variations are small enough to assume that the free-field correction of a typical microphone of such a type is valid for all microphones of the same type within a stated uncertainty. In any case, the determination of the free-field correction involves measurement of the pressure and free-field sensitivities of the microphones.

Alternatively, the free-field correction can be determined using the expression ${ }^{1}$

$$
C_{\mathrm{ff}}=20 \log _{10}\left\{\int\left(p(r) / p_{0}\right) v(r) r d r / \int v(r) r d r\right\},
$$

where $p(r)$ is the pressure on the membrane as a function of the radius $r, v(r)$ is the velocity of the membrane as a function of $r$, and $p_{0}$ is the undisturbed incident pressure. The pressure on the membrane can be determined numerically when the velocity is known. The calculation is carried out using an iterative procedure that also involves estimating the acoustic impedance of the microphone by means of any available method.

\section{Directivity index}

The random-incidence sensitivity of a microphone can be determined as the sum of the pressure sensitivity and the random-incidence correction. The random-incidence response is the average of the free-field response of the microphone to incoherent plane waves coming from all directions, and thus it has similar properties as the free-field correction. The random-incidence correction can also be determined from the directivity index and the free-field response. In general, the directivity factor, $Q$, at the frequency $f$ is defined as

$$
Q(f)=\frac{4 \pi\left|H\left(f, \theta_{0}, \phi_{0}\right)\right|^{2}}{\int_{0}^{2 \pi} \int_{0}^{\pi}|H(f, \theta, \phi)|^{2} \sin \theta d \theta d \phi},
$$

where $H(f, \theta, \phi)$ is the frequency response at the angles $\theta$ and $\phi$. The index 0 indicates the axial direction. ${ }^{19}$ Assuming that the microphone is axi-symmetric and substituting the integral by a discrete series, Eq. (6) simplifies to

$$
Q(f)=\frac{2\left|H\left(f, \theta_{0}\right)\right|^{2}}{\sum_{n=1}^{\pi / \Delta \theta}|H(f, \theta)|^{2} \sin \theta_{n} \Delta \theta} .
$$

The directivity index, $D$, is the directivity factor expressed in logarithmic fashion, i.e.,

$$
D=10 \log Q \text {. }
$$

\section{EXPERIMENTAL SETUP}

The velocity of the membranes of various microphones has been measured using a laser vibrometer, Polytech PDV100. The microphone was used as a transmitter, and its membrane was driven using a reciprocity apparatus, Brüel \& Kjær (B\&K) type 5998. The voltage on the terminals of the reference impedance on the transmitter unit, $B \& K$ type ZE0796, and the output of the vibrometer was measured using a B\&K "PULSE" analyzer. Figure 2 shows a block diagram of the measurement setup, and Fig. 3 shows a picture of the vibrometer and the microphone mounted on the positioning rig.

The signal used for driving the microphone was pseudorandom noise with a bandwidth of $25.6 \mathrm{kHz}$ and $6400 \mathrm{spec}-$ tral lines. The laser vibrometer can measure up to $24 \mathrm{kHz}$. Although several types of microphones were measured, only results for 1-in. and 0.5-in. LS microphones (LS1 and LS2, respectively) are presented in what follows.

\section{BEM MODELING}

An open source implementation of BEM has been used in the calculations. Details of the formulation can be found elsewhere, ${ }^{1}$ but summarizing, such a formulation relates a possible incident wave $p^{I}$ and the pressure $p$ and velocity $v$ at the point $Q$ on the surface $S$ of a body to the pressure at the external point $P$,

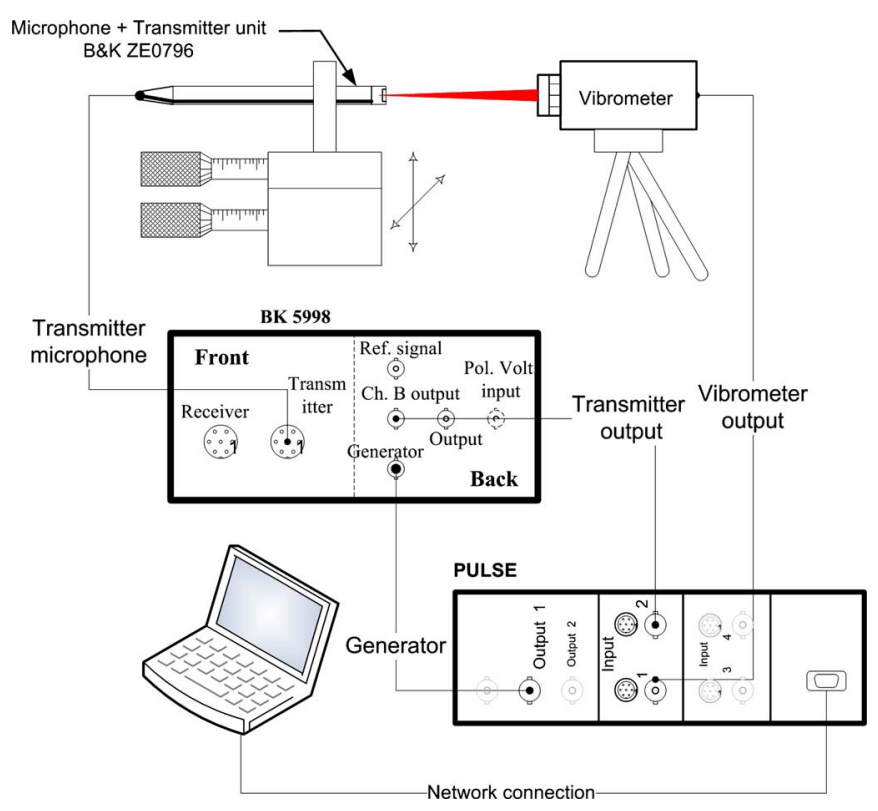

FIG. 2. (Color online) Block diagram of the measurement system. 


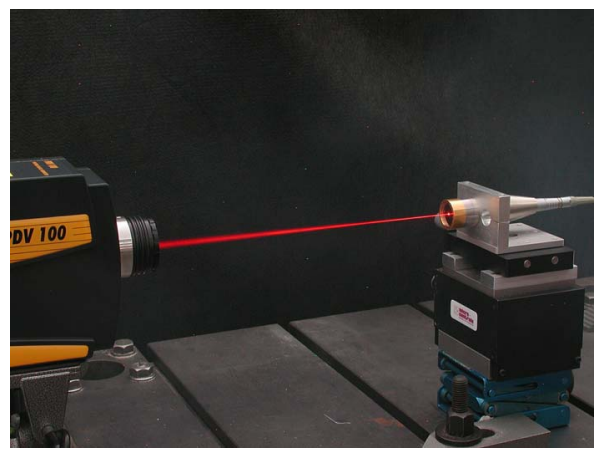

FIG. 3. (Color online) Measurement setup. The laser beam measured the velocity at a point on the membrane.

$$
\begin{aligned}
C(P) p(P)= & \int_{L} \int_{\theta}\left(p(Q) \frac{\partial G(R)}{\partial n}+j k z_{0} v(Q) G(R)\right) \\
& \times d \theta \rho(Q) d L(Q)+4 \pi p^{I}(P),
\end{aligned}
$$

where $G(R)=e^{-j k R} / R$ is the free space Green's function, $R$ is the distance between the points $P$ and $Q, z_{0}$ is the characteristic impedance of air $(\rho c)$, and $C(P)$ is the solid angle seen from $P$. In the axi-symmetric case, the velocity and pressure are independent of the rotation angle, $\phi$. However, it is possible to introduce non-axi-symmetric boundary conditions if the pressure and velocity are expanded in cosine series. In this case, only the axi-symmetrical expansion (zeroth term) has been used. The methodology proposed in this paper is to define $v(Q)$ in Eq. (9) as the measured velocity of the membrane instead of assuming an analytical form.

The geometry used in the BEM calculations is shown in Fig. 4. The ideal, semi-infinite rod was approximated by a cylindrical rod with a length of $60 \mathrm{~cm}$ and a hemispherical back-end. The termination of the rod inevitably gives rise to reflections, but because of the length of the rod the reflections can be expected that to have a negligible influence.

The BEM calculations were used for determining quantities such as the acoustic center, the free-field correction, and the directivity index. The acoustic center has an asymptotic behavior at low frequencies, and the directivity index and the free-field correction tend to zero at low fre-

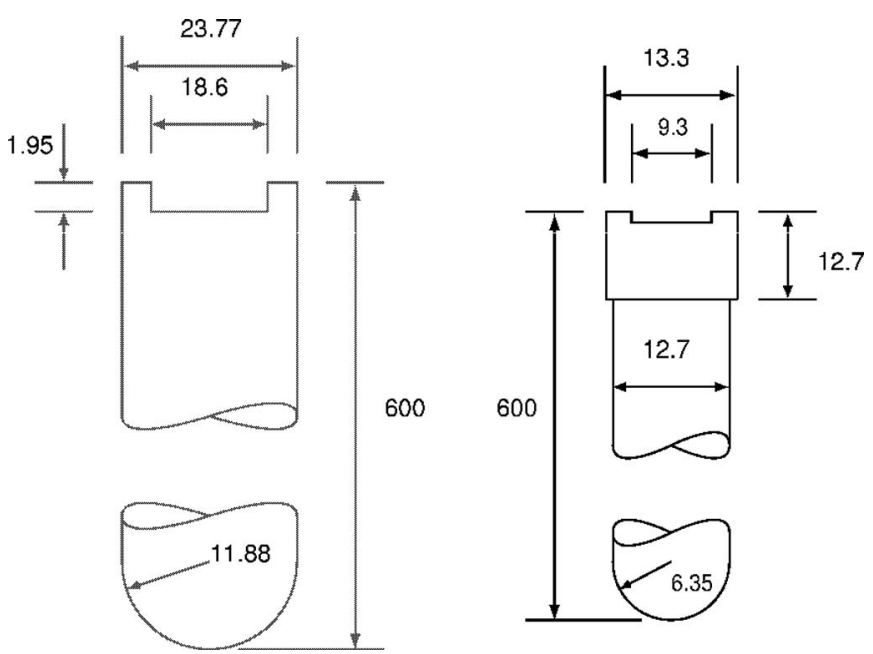

FIG. 4. Geometry of LS1 and LS2 microphones used in the simulations. quencies. Therefore, there is no need for calculations, say, below $1 \mathrm{kHz}$ for LS1 microphones. Moreover, the experimental estimates to be compared with the results from the hybrid method have a comparable lower frequency limit. On the other hand, the laser vibrometer can only measure up to $24 \mathrm{kHz}$, which sets the high frequency limit. Hence, the frequency range used in the calculations was from 1 to $24 \mathrm{kHz}$ for LS1 microphones and from 2 to $24 \mathrm{kHz}$ for LS2 microphones. The size of the smallest element in the axisymmetric mesh was 2.5 and $1.5 \mathrm{~mm}$ for LS1 and LS2 microphones, respectively. Thus, there were at least six elements per wavelength at the highest frequency.

In order to avoid the non-uniqueness problem, a random CHIEF point was added in the interior of the geometry as described in Ref. 20, and the calculations were checked by determining the condition numbers of the BEM matrices and by repeating calculations with small frequency shifts. ${ }^{21}$

Depending on the quantity to be determined, the microphone acts as receiver or as a source. When the microphone acts as a source, the radiation problem is solved by assigning the measured velocity to the membrane of the microphone. In the scattering problem, the structural coupling between the membrane and the scattered sound field is solved using an iterative procedure.

\section{RESULTS AND DISCUSSION}

\section{A. Movement of the membrane}

Figures 5 and 6 show the measured velocity of the membrane of two different types of microphones at different frequencies. It can be seen in Fig. 5 that the shape of the movement of the membrane of an LS1 microphone is similar to a parabola at frequencies below $5 \mathrm{kHz}$. Above this frequency and around the resonance frequency, the shape deviates from the assumed parabola, and the deviations become more obvious the higher the frequency. From $14 \mathrm{kHz}$ and upward, the center of the membrane flattens and no longer looks like a parabola nor like any other simple analytical shape. It is apparent that above $20 \mathrm{kHz}$, the center of the membrane does not move as much at a rim between the center and the fixed perimeter of the membrane. The velocity profiles are the result of the interaction between the membrane and the backplate of the microphone. The positions of the maxima coincide with the position of the holes and the recess on the backplate.

The movement of the membrane of an LS2 microphone shows a different behavior (see Fig. 6). It can be seen that the shape is more regular in the same frequency interval even around the resonance frequency (approximately $18 \mathrm{kHz}$ ). Only above the resonance frequency does the shape seem to flatten slightly. It can also be noticed that in both cases there is a phase delay at the center of the membrane with respect to the rim. This makes the movement of the membrane even more complex and difficult to emulate using simple analytical shapes. Thus, it seems to be difficult to make any a priori assumption of the movement of the membrane above the resonance frequency of the microphones. 


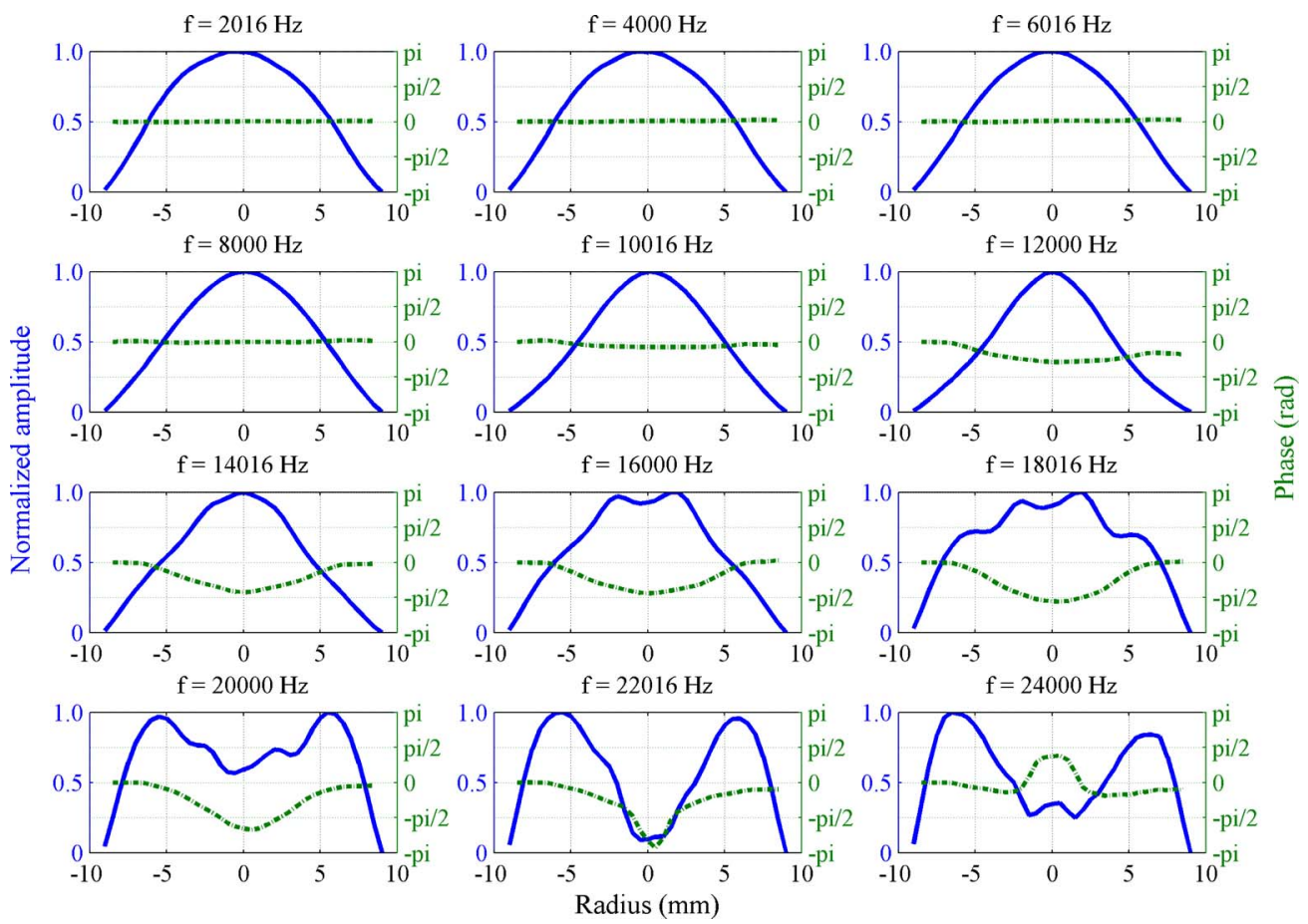

FIG. 5. (Color online) Velocity of the membrane of an LS1 microphone measured with a laser vibrometer at several frequencies. Solid line: normalized amplitude; dash-dotted line: phase.

\section{B. Pressure sensitivity}

There is no need for BEM calculations in determining the pressure sensitivity. Determining the ratio of the volume velocity to the current in Eq. (2) simply requires integrating the velocity over the membrane. Figures 7(a) and 7(b) show the normalized pressure sensitivity of an LS1 and an LS2 microphone compared with the experimental response obtained using the reciprocity technique.
At high frequencies, around and above the resonance, the difference between the responses determined by reciprocity calibration and by Eq. (2) is caused by the different load of the radiation impedance described by Eq. (2). In the vibrometer measurements, the radiation impedance is that of a microphone radiating to the open, whereas in the case of reciprocity calibration, the radiation impedance is the input impedance of a coupler terminated by a finite impedance at
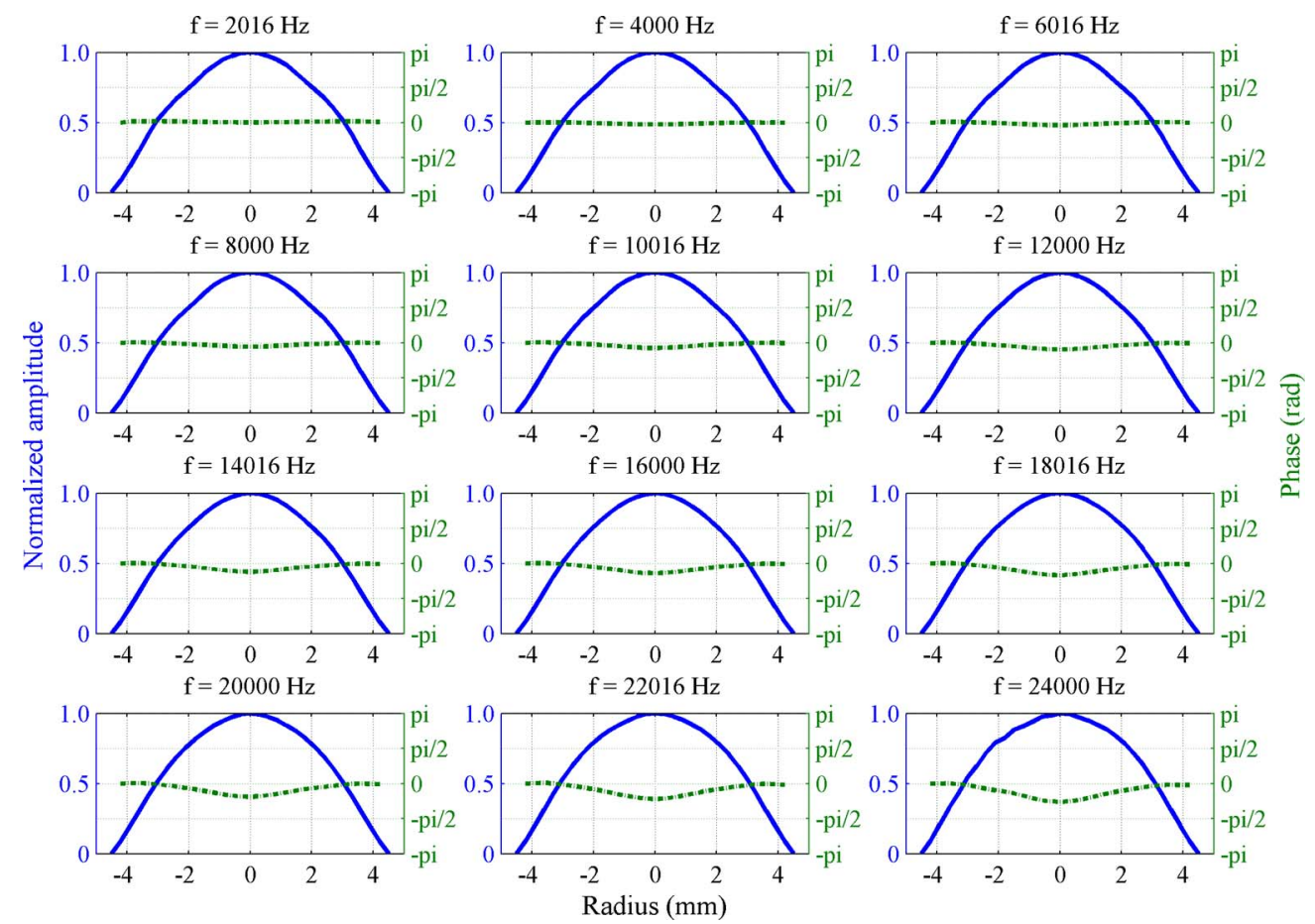

FIG. 6. (Color online) Velocity of the membrane of an LS2 microphone measured with a laser vibrometer at several frequencies. Solid line: normalized amplitude; dash-dotted line: phase. 

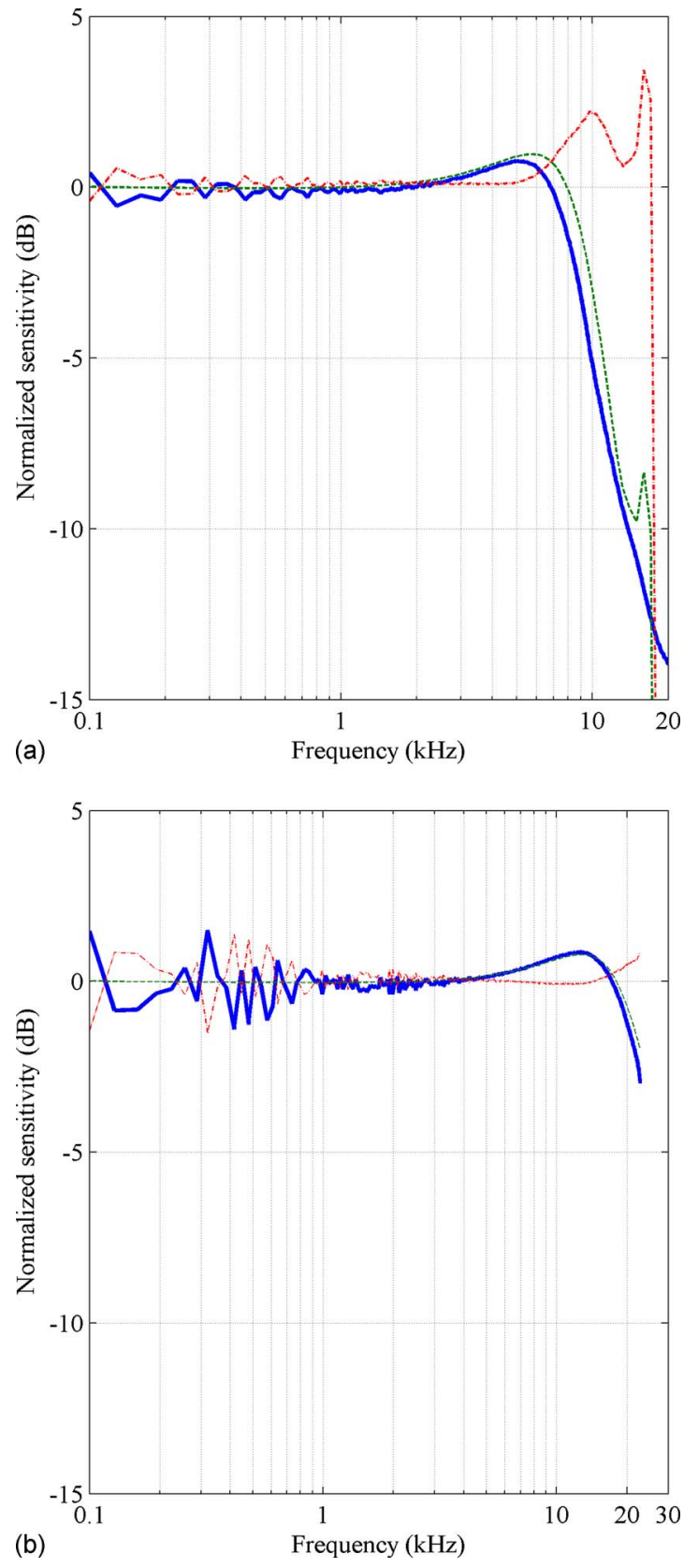

FIG. 7. (Color online) Normalized pressure response of (a) an LS1 microphone and (b) an LS2 microphone. Solid line: estimate from measurements of the velocity of the membrane; dashed line: estimate obtained from reciprocity calibration; dash-dotted line: difference between estimates.

the opposite end. The radiation impedance that loads the membrane in the vibrometer method is very similar to the radiation impedance that loads the membrane in electrostatic actuator measurements, and therefore a similar difference between reciprocity calibration and the hybrid method and between reciprocity calibration and actuator calibration can be expected. The difference seen in Figs. 7(a) and 7(b) agrees quite well with results observed in the literature for the difference between reciprocity and electrostatic actuator. ${ }^{17}$ However, there is a small peak at about $16 \mathrm{kHz}$ for the LS1 microphones that does not occur in the estimate from velocity measurements. This may be caused by the resonances of longitudinal modes in the couplers used in reciprocity measurements.
In the LS1, and in particular in the LS2 results, erratic sawtooth variations occur below $1 \mathrm{kHz}$. These variations may have been caused by insufficient averaging. Below 1 $\mathrm{kHz}$, the microphones are very poor radiators of sound partly because the amplitude of the displacement of the membrane is very small, and the measurements may well have been affected by extraneous noise. It seems likely that an optimization of the measurement technique could improve the results.

\section{Acoustic center}

Figure 8 shows the acoustic center of an LS1 microphone determined from numerical BEM calculations using a parabolic function, a Bessel-like function, and the measured velocity distribution. The results are compared with data obtained experimentally from reciprocity measurements. It can be seen that the agreement between measured data and the $\mathrm{BEM}$ calculations using the measured velocity distribution is very good at any frequency above $2 \mathrm{kHz}$. The bump at 1.5 $\mathrm{kHz}$ in the estimate determined from measurements of the transfer function between two microphones is caused by the application of the time-selective procedure as discussed above. This suggests that the sound field calculated from the measured velocity distribution is more accurate than calculations based on any assumed distribution, in particular at high frequencies.

\section{Free-field correction}

Figure 9 shows the free-field correction of LS1 microphones determined with the hybrid method and from combined free-field and pressure reciprocity calibrations. The agreement between the reciprocity result and the estimate obtained with the hybrid method is not very good around and above the resonance frequency (about $8 \mathrm{kHz}$ ). The reason for this may be that in order to determine the correction an iterative BEM calculation procedure that requires knowledge of the acoustic impedance of the membrane of the micro-

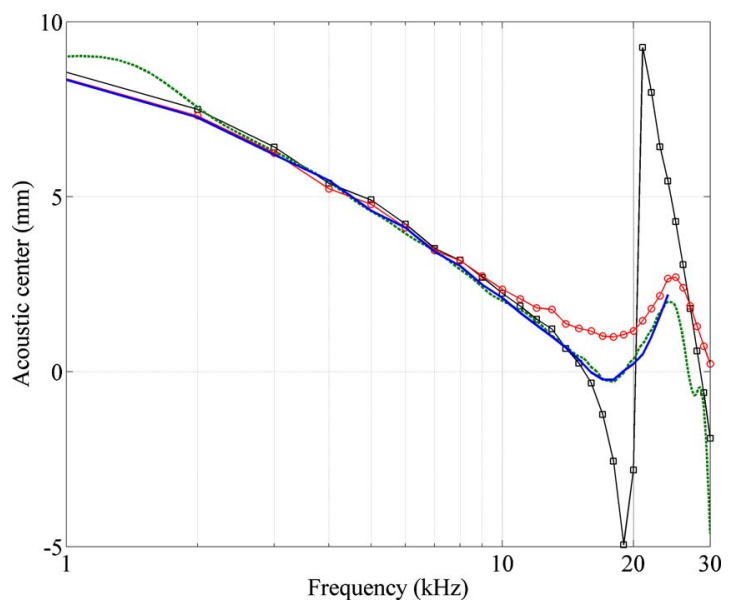

FIG. 8. (Color online) Acoustic center of LS1 microphones. Solid line: estimate from the hybrid method; dashed line: estimate determined from measurements of the transfer function between two microphones; line with square markers: numerical estimate assuming a Bessel-like movement; line with circular markers: numerical estimate obtained assuming a uniform velocity distribution. 


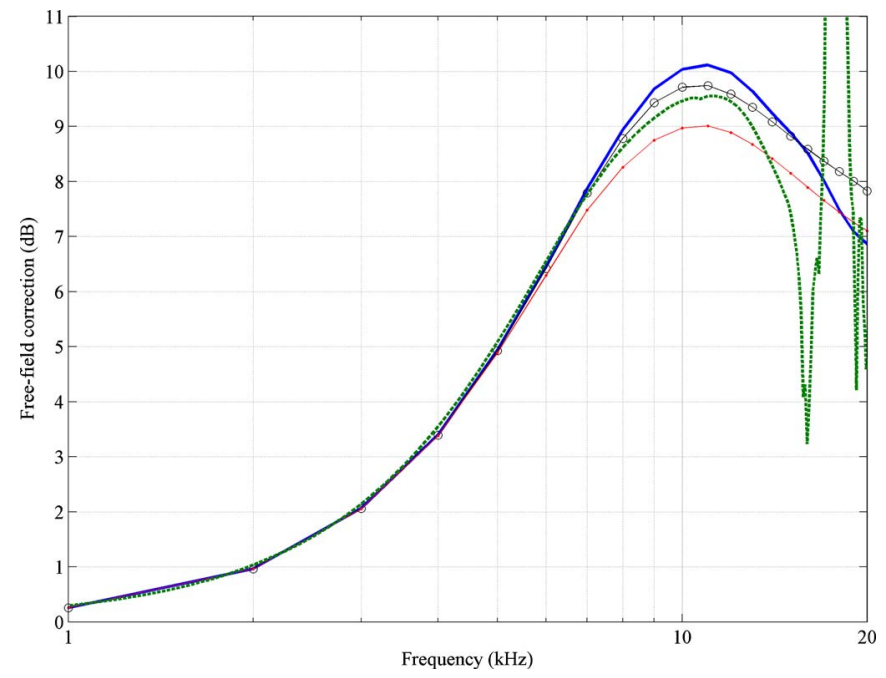

FIG. 9. (Color online) Free-field correction of LS1 microphones. Solid line: estimate from the hybrid method; dashed line: estimate determined from free-field and pressure reciprocity; line with circular markers: numerical estimate assuming parabolic movement; line with dot markers: numerical estimate assuming uniform movement.

phone has been be used. In this case, a lumped-parameter approximation of the acoustic impedance was used. ${ }^{1}$ This approximation is only accurate at frequencies below the resonance frequency, and therefore it is expected that the hybrid method should give less accurate results at frequencies near and above the resonance. Besides, as mentioned above, at frequencies above $15 \mathrm{kHz}$ the pressure sensitivity determined from reciprocity, and thus the free-field correction, is no longer reliable due to longitudinal and radial resonances in the couplers.

\section{E. Directivity index}

Figure 10 shows the directivity index of LS1 and LS2 microphones. It is evident that the directivity index calculated from the measured velocity distribution follows the experimental estimate better than calculations based on an assumed distribution. This is particularly clear for the case of the LS1 microphone, in which the experimental index shows a change in slope at around $15 \mathrm{kHz}$. This behavior cannot be reproduced when using a Bessel movement in the simulations.

In the case of the LS2 microphones, the difference between the results of the experimental and the hybrid method coincide very well up to $24 \mathrm{kHz}$ and with the numerical calculations using a Bessel-like movement. This is not unexpected because the resonance frequency of the microphone is about $22 \mathrm{kHz}$, and the actual movement of the membrane is "well-behaved" and still resembles a Bessel function; the same can be said of LS1 microphones at frequencies around and below the resonance frequency (about $8 \mathrm{kHz}$ for LS1 microphones). However, nothing can be said for LS2 microphones at frequencies much higher than resonance because these frequencies are outside the measurement range of the laser vibrometer.

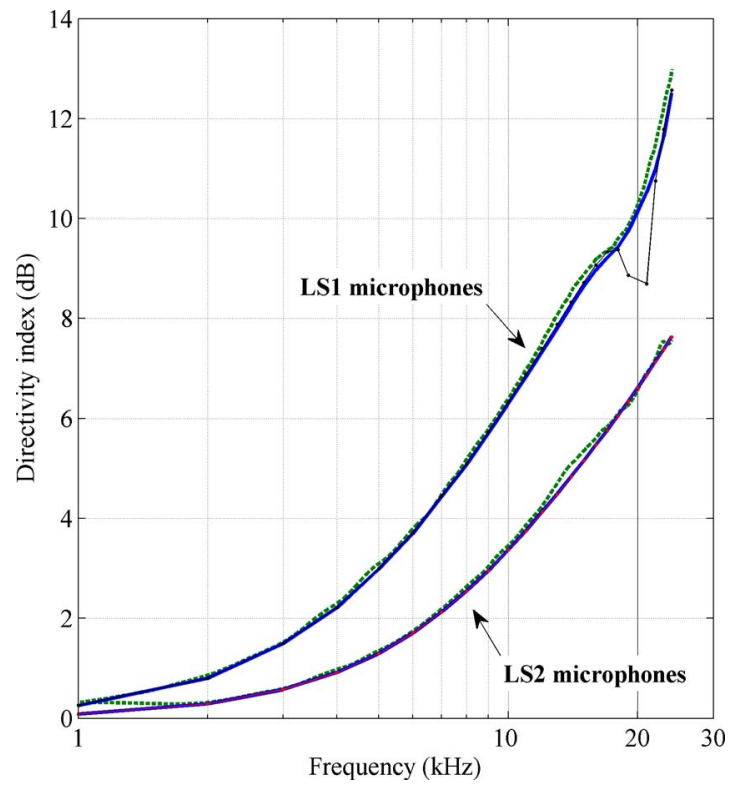

FIG. 10. (Color online) Directivity index of LS1 and LS2 microphones determined experimentally and with the hybrid method. The arrows point to the results for each type of microphone. Solid line: hybrid method; dashed line: estimate determined using the random-incidence technique; line with solid circular markers: numerical estimate assuming Bessel-like movement.

\section{CONCLUSIONS}

The velocity distributions of the membranes of different types of microphones measured with a laser vibrometer have been found to be fairly complicated and demonstrate that no general assumption can be made for the behavior of all microphones. Preliminary results of the pressure sensitivity, the acoustic center, the free-field correction, and the directivity index obtained by a hybrid method that combines the measured velocity distributions with calculations with the BEM are, in general, in good agreement with experimental results obtained by traditional methods where the quantities are determined directly. It can therefore be concluded that the hybrid method can be used for validating new experimental setups. Furthermore, the hybrid method can be used in production environments to check the responses of a prototype microphone without the need of a complete calibration setup, and it is potentially useful for calibrating non-standard microphones. However, the hybrid method is not a substitute of an individual calibration of a particular transducer.

\section{ACKNOWLEDGMENT}

The investigation presented in this paper was made as a part of a project carried out with support from the Danish Agency for Technology and Production (FTP).

${ }^{1} \mathrm{P}$. M. Juhl, "A numerical investigation of standard condenser microphones," J. Sound Vib. 177, 433-446 (1994).

${ }^{2}$ A. J. Zuckerwar, "Theoretical response of condenser microphones," J. Acoust. Soc. Am. 64, 1278-1285 (1978).

${ }^{3}$ S. Barrera-Figueroa, K. Rasmussen, and F. Jacobsen, "The acoustic center of laboratory standard microphones," J. Acoust. Soc. Am. 120, 2668-2675 (2006).

${ }^{4}$ S. Barrera-Figueroa, K. Rasmussen, and F. Jacobsen, "On experimental determination of the free-field correction of laboratory standard microphones at normal incidence," Metrologia 44, 57-63 (2007).

${ }^{5}$ S. Barrera-Figueroa, K. Rasmussen, and F. Jacobsen, "On experimental 
determination of the random-incidence response of microphones," J. Acoust. Soc. Am. 121, 2628-2636 (2007).

${ }^{6} \mathrm{X}$. Bao and Y. Kagawa, "A simulation of condenser microphones in free field by boundary element approach," J. Sound Vib. 119, 327-337 (1987).

${ }^{7}$ V. Cutanda-Henríquez, "Numerical transducer modeling," Ph.D. thesis, Ørsted-DTU, Technical University of Denmark (DTU), Denmark (2002).

${ }^{8}$ R. S. Grinnip III, "Advanced simulation of a condenser microphone capsule," J. Audio Eng. Soc. 54, 157-166 (2006).

${ }^{9} \mathrm{G}$. Behler and M. Vorländer, "Reciprocal measurements on condenser microphones for quality control and absolute calibration," Acta Acust. 90, 152-160 (2004).

${ }^{10}$ W. R. MacLean, "Absolute measurement of sound without a primary standard," J. Acoust. Soc. Am. 12, 140-146 (1940).

${ }^{11}$ A. D. Pierce, Acoustics: An Introduction to Its Physical Principles and Applications (Acoustical Society of America, New York, 1994), Chap. 4.10, pp. 199-203.

12“"Measurement microphone-Part 3: Primary method for free-field calibration of laboratory standard microphones by the reciprocity technique," IEC International Standard 61094-3 (International Electrotechnical Commission, Geneve, Switzerland, 1995).

${ }^{13} \mathrm{~W}$. Wathen-Dunn, "On the reciprocity free-field calibration of microphones," J. Acoust. Soc. Am. 21, 542-546 (1949).
${ }^{14}$ I. Rudnick and M. N. Stein, "Reciprocity free-field calibration of microphones to $100 \mathrm{kc}$ in air," J. Acoust. Soc. Am. 20, 818-825 (1948).

${ }^{15}$ K. Rasmussen, "Acoustic centres of condenser microphones," Report No. 5, The Acoustics Laboratory, Technical University of Denmark, 1971.

${ }^{16}$ D. Rodrigues, J. N. Durocher, M. Bruneau, and A. M. Bruneau, "Freefield calibration of microphones: Theoretical and experimental determination of the acoustic center (A)," J. Acoust. Soc. Am. 123, 3847 (2008).

${ }^{17} \mathrm{G}$. Rasmussen, "The free field and pressure calibration of condenser microphones using electrostatic actuator," Brüel \& Kjær Technical Review 2, 3-7 (1969).

18“"Measurement microphones-Part 7: Values for the difference between free-field and pressure sensitivity levels of laboratory standard microphones," IEC Technical Specification 61094-7, 1st ed. (International Electrotechnical Commission, Geneve, Switzerland, 2006).

${ }^{19}$ L. L. Beranek, Acoustical Measurements (American Institute of Physics, Melville, NY, 1988).

${ }^{20} \mathrm{H}$. A. Schenck, "Improved integral formulation for acoustic radiation problems," J. Acoust. Soc. Am. 44, 41-58 (1968).

${ }^{21}$ P. M. Juhl, F. Jacobsen, V. Cutanda, and S. Quiros-Alpera, "On the nonuniqueness problem in a 2-D half-space BEM formulation," in Proceedings of the Ninth International Congress on Sound and Vibration, Orlando, FL (2002). 